\title{
Big Five Personality Characteristics and Adherence to Clinic-Based Rehabilitation Activities After ACL Surgery: A Prospective Analysis
}

\author{
R.C. Hilliard ${ }^{1}$, B.W. Brewer ${ }^{*}, 2$, A.E. Cornelius ${ }^{2}$ and J.L. Van Raalte ${ }^{2}$ \\ ${ }^{1}$ Kalamazoo College, 1200 Academy Street, Kalamazoo, MI 49006, USA \\ ${ }^{2}$ Springfield College, 263 Alden Street, Springfield, MA 01109, USA
}

\begin{abstract}
Purpose: A prospective, longitudinal study was conducted to examine Big Five personality characteristics as predictors of adherence to clinic-based rehabilitation activities following anterior cruciate ligament (ACL) reconstruction surgery.

Methods: Participants (72 men, 36 women) completed a questionnaire assessing Big Five personality dimensions prior to surgery. For the first 7 weeks after surgery, participants' rehabilitation session attendance was recorded and rehabilitation professionals rated participants' adherence during rehabilitation sessions.

Results: Results of multiple regression analyses indicated that the 5 personality factors explained 9 percent of the variance in attendance and 16 percent of the variance in adherence ratings, that agreeableness was a significant positive predictor of attendance, and that conscientiousness and openness to experience were significant positive predictors of adherence ratings.

Conclusion: As a potential contributor to adherence, personality warrants consideration when implementing rehabilitation programs after ACL surgery.
\end{abstract}

Keywords: Attendance, big five, compliance, knee, physical therapy, psychology.

\section{INTRODUCTION}

Rehabilitation is a common component in the treatment of sport injuries. As with other medical conditions, for which more than 200 factors predictive of adherence to treatment have been identified [1], treatment adherence is an issue of concern in sport injury rehabilitation. Across various sport injuries and indices of adherence to sport injury rehabilitation, adherence rates have ranged from 40 to 91 percent in empirical studies [2]. Evidence of a positive correlation between adherence to sport injury rehabilitation and treatment outcome has been documented (for a review, see [3]), highlighting the potential importance of sport injury rehabilitation adherence.

In the integrated model of psychological response to sport injury [4], rehabilitation behaviors such as adherence to treatment are described as being influenced by personal, situational, cognitive, and emotional factors. Although positive correlations have been observed between several personal factors (e.g., self-motivation, pain tolerance, toughmindedness) and sport injury rehabilitation adherence [3], little attention has been given to major dimensions of personality as possible predictors of adherence to sport injury rehabilitation. Specifically, dimensions of the Big Five model of personality [5] - conscientiousness, agreeableness, neuroticism, openness to experience, and

*Address correspondence to this author at the Springfield College, 263 Alden Street, Springfield, MA 01109, USA; Tel: +1-413-748-3696;

Fax: +1-413-748-3854; E-mail: bbrewer@springfieldcollege.edu extraversion - have not been considered in relation to sport injury rehabilitation adherence.

Research on other health behaviors provides a compelling rationale for examining the relationship of Big Five personality dimensions to sport injury rehabilitation adherence. Across studies of adherence to exercise regimens, medication regimens, and various "wellness behaviors," positive associations have been found between adherence and conscientiousness [6-9], agreeableness [6], and extraversion $[6,8,10-12]$. Negative associations have been documented between neuroticism and adherence [6, 8, 11]. Accordingly, the present study was designed to assess the extent to which the findings regarding the relationship between these four Big Five personality dimensions and adherence to other health behaviors generalize to adherence to sport injury rehabilitation activities.

To minimize variability in rehabilitation protocols across participants, a single injury type - an acute rupture of the ligament that helps to provide stability to the knee, the anterior cruciate ligament (ACL) — was examined. Typically sustained during sport participation, an acute rupture of the ACL is a common and debilitating injury that is often treated with surgical reconstruction and requires an extended period of postoperative rehabilitation. Adherence to rehabilitation regimens is considered particularly important because ACL surgery is among the medical procedures for which research has shown that better adherence to rehabilitation is associated with more favorable treatment outcomes [3]. Based on previous research [6-12], it was hypothesized that conscientiousness, agreeableness, and extraversion would be 
positively associated and neuroticism would be negatively associated with adherence to clinic-based rehabilitation following ACL surgery. No hypotheses were advanced for the fifth Big Five dimension-openness to experience-in light of the lack of previously reported correlations between this dimension and adherence.

\section{METHODS}

\section{Participants}

Participants were selected on a consecutive patient basis from among those who had an ACL reconstruction with one of three orthopedic surgeons affiliated with the study, planned to do their postoperative rehabilitation at one of three physical therapy clinics affiliated with the study, and consented to participate in the study. A total of 108 individuals (72 men, 36 women) participated in the study. One person who met the criteria decided against participation. The mean age of participants was 29.38 $(\mathrm{SD}=9.93$, range $=14-54)$ years. Participants were predominantly (90\%) White, not of Hispanic origin.

\section{Rehabilitation Protocol}

The program for rehabilitation after ACL surgery that Shelbourne and his associates designed $[13,14]$ and later revised [15] was implemented by the physical therapists in this study. This accelerated program concentrates on knee extension, laxity, gait, and strength, and advancement through the program is based on the knee's functional ability rather than a prescribed time schedule. Under this program, patients are typically asked to complete multiple clinic-based rehabilitation sessions each week for several months.

\section{Measures}

Information regarding the demographic characteristics, injury, adherence, and personality of participants was assessed. Demographic and injury-related information was assessed with a questionnaire that had items addressing the age, gender, race/ethnicity, and injury date of participants.

Adherence was measured two ways. First, rehabilitation session attendance was recorded. The ratio of the number of sessions attended to the number of sessions scheduled was computed. Second, the rehabilitation practitioners overseeing the clinic appointments of participants completed the Sport Injury Rehabilitation Adherence Scale (SIRAS) [16] at the end of each rehabilitation session. The SIRAS is a questionnaire with 3 items on which clinicians rate the intensity of patients' completion of rehabilitation exercises, the frequency of following the clinicians' instructions and advice, and openness to modifications of the treatment regimen during rehabilitation appointments. Clinicians rate patients on the SIRAS using 5-point Likert-type scales with anchors of minimum effort/maximum effort, never/always, and very unreceptive/very receptive, respectively, for the three items. A Cronbach's alpha coefficient of 0.81 and a test-retest reliability (intraclass) coefficient of 0.77 over a one-week period have been documented for the SIRAS [16]. Support for the scale's construct validity and interrater agreement have also been obtained [17, 18]. An average SIRAS score across rehabilitation sessions was calculated for all participants.
Personality was assessed with the NEO-Five Factor Inventory-S (NEO-FFI-S) [19]. This questionnaire has 60 items and measures the Big Five personality dimensions using a 5-point Likert-type scale with anchors of strongly disagree and strongly agree. Costa and $\mathrm{McCrae}$ presented evidence in support of the internal consistency (alpha coefficients ranging from $\alpha=.86$ to .92 ), test-retest reliability over a 3-month period (reliability coefficients ranging from .75 to .83 ), and construct validity of the NEO-FFI-S [19].

\section{Procedure}

Approval to conduct the research was granted by the institutional review board at Springfield College. Participants who fulfilled the selection criteria for the study were recruited from the practices of the orthopedic surgeons involved with the study. Patients who agreed to participate in the study received a description of the purpose and procedures of the study from a research assistant. One week before their surgery, at a preoperative visit, participants (and parent or guardian if necessary) completed a written informed consent form and provided contact information. Participants then completed the demographic and injuryrelated questionnaire and the NEO-FFI-S.

Measurement of adherence to clinic-based rehabilitation activities began with the first postoperative session of physical therapy following surgery and continued for the first seven weeks of rehabilitation. A research assistant recorded participants' attendance at rehabilitation sessions and collected SIRAS ratings from the rehabilitation practitioner who supervised the treatment of the participants on those days.

\section{Data Analysis}

Correlation and hierarchical regression analyses were conducted to explore associations between the Big Five personality dimensions and adherence to clinic-based ACL rehabilitation activities. In the first hierarchical regression analysis, attendance was the criterion variable. Age and sex were entered on the first step as control variables and the Big Five personality factors were entered on the second step. The second hierarchical regression analysis was identical to the first with the exception that SIRAS scores constituted the criterion variable.

\section{RESULTS}

Complete data sets were obtained for 104 of the 108 participants. Means and standard deviations of the personality and adherence measures are displayed in Table $\mathbf{1}$. Relative to the norms provided for the NEO-FFI-S [19], the mean scores for all of the Big Five personality factors were in the "average" range, with exception of that for extraversion, which was in the "high" range. Intercorrelations among the personality and adherence measures are presented in Table 2. Only agreeableness was significantly correlated with attendance and only conscientiousness was significantly correlated with SIRAS scores.

In the first hierarchical regression analysis, the Big Five personality factors predicted a significant incremental proportion of the variance in attendance over and above age and sex, $R_{\text {change }}^{2}=.09, F(5,96)=2.43, p=.04$. As shown in 
Table 3, agreeableness emerged as a significant predictor of attendance $(\beta=.27, p=.01)$. Participants with high agreeableness scores attended a higher percentage of their scheduled rehabilitation appointments than participants with low agreeableness scores.

Table 1. Descriptive statistics of the sample.

\begin{tabular}{|c|c|c|c|}
\hline & $\boldsymbol{M}$ & $\boldsymbol{S D}$ & $\boldsymbol{N}$ \\
\hline \hline Attendance & .91 & .14 & 107 \\
\hline SIRAS & 13.73 & .86 & 107 \\
\hline Age & 29.38 & 9.93 & 107 \\
\hline Neuroticism & 17.11 & 7.10 & 107 \\
\hline Extraversion & 31.84 & 5.49 & 108 \\
\hline Openness to experience & 25.81 & 5.19 & 107 \\
\hline Agreeableness & 31.12 & 5.29 & 108 \\
\hline Conscientiousness & 34.14 & 6.43 & 108 \\
\hline
\end{tabular}

Note. Missing data resulted in a sample size $<108$ for some variables.

In the second hierarchical regression analysis, the Big

Five personality factors accounted for a significant incremental proportion of the variance in practitioner ratings of adherence over and above age and sex, $R_{\text {change }}^{2}=.16$, $F(5,96)=3.07, p=.006$. Openness to experience $(\beta=.24$, $p=.01)$ and conscienti-ousness $(\beta=.35, p<.001)$ were significant predictors of SIRAS scores, with positive associations obtained for both factors.

\section{DISCUSSION}

The results of this investigation are in line with both theory and past research. As has typically been the case in investigations of individuals undergoing rehabilitation of sport-related injuries, most of the participants attended the vast majority of their scheduled appointments [2], perhaps reflecting strong motivation to return to sport involvement. As posited in the integrated model of psychological response to sport injury [4], personal factors (in the form of Big Five personality characteristics) were predictive of behavioral responses to sport injury in the form of adherence to rehabilitation. Conscientiousness, agreeableness, and openness to experience join self-motivation, pain tolerance, and toughmindedness as personal factors correlated with sport injury rehabilitation adherence [3]. The results augment past investigations in which conscientiousness and agreeableness were positively correlated with other health behavior regimens $[6-9,11]$. It is not surprising that

Table 2. Intercorrelations among personality and adherence variables.

\begin{tabular}{|c|c|c|c|c|c|c|c|}
\hline & $\mathbf{N}$ & $\mathbf{E}$ & $\mathbf{O}$ & $\mathbf{A}$ & C & Att & SIRAS \\
\hline $\mathrm{N}$ & - & $-.32 * *$ & .00 & $-.23 *$ & $-.22 *$ & -.08 & -.17 \\
\hline $\mathrm{E}$ & & - & .01 & $.31^{*}$ & $.34 * *$ & .19 & .10 \\
\hline $\mathrm{O}$ & & & - & .01 & -.13 & .15 & .19 \\
\hline A & & & & - & $.24^{*}$ & $.31 * *$ & .13 \\
\hline $\mathrm{C}$ & & & & & - & .08 & $.33 * *$ \\
\hline Att & & & & & & - & $.24 *$ \\
\hline SIRAS & & & & & & & - \\
\hline
\end{tabular}

Note. $\mathrm{N}=$ neuroticism, $\mathrm{E}=$ extraversion, $\mathrm{O}=$ openness to experience, $\mathrm{A}=$ agreeableness, $\mathrm{C}=$ conscientiousness, Att $=$ attendance, SIRAS $=$ mean of total SIRAS scores. ${ }^{*} p<.05$.

$* * p<.01$.

Table 3. Results of hierarchical regression analysis predicting attendance.

\begin{tabular}{|c|c|c|c|c|c|}
\hline Predictor & Total $R^{2}$ & $F$ & $F_{\text {change }}$ & $\beta$ & $p$ \\
\hline Step 1 & .02 & 1.13 & 1.13 & & \\
\hline Sex & & & & -.14 & .15 \\
\hline Step 2 & .11 & $2.08^{*}$ & $2.43^{*}$ & & \\
\hline Extraversion & & & & .11 & .34 \\
\hline Openness & & & & .15 & .12 \\
\hline Agreeableness & & & & .27 & $.02 *$ \\
\hline Conscientiousness & & & & -.02 & .88 \\
\hline
\end{tabular}


Table 4. Results of hierarchical regression analysis predicting SIRAS scores.

\begin{tabular}{|c|c|c|c|c|c|}
\hline Predictor & Total $R^{2}$ & $\boldsymbol{F}$ & $F_{\text {change }}$ & $\beta$ & $p$ \\
\hline Step 1 & .01 & .52 & .52 & & \\
\hline Sex & & & & -.01 & .90 \\
\hline Step 2 & .17 & $3.07 *$ & $4.06^{*}$ & & \\
\hline Extraversion & & & & -.04 & .68 \\
\hline Openness & & & & .24 & $.01 *$ \\
\hline Agreeableness & & & & .05 & .66 \\
\hline Conscientiousness & & & & .35 & $.001 * *$ \\
\hline
\end{tabular}

participants high in conscientiousness, a personality dimension characterized by hard work, persistence, and perseverance [20-23], would be rated as more adherent during rehabilitation sessions than those low in conscientiousness. Similarly, the tendency of participants high on agreeableness to attend a greater percentage of their rehabilitation appointments than those low on agreeableness aligns with the compliant attitudes characteristic of the agreeableness trait [21].

The positive association between openness to experience and ratings of adherence during rehabilitation appointments was not predicted given the dearth of previous research support for such a relationship. A potential interpretation of this result is that with its rapid progression and varied activities, the accelerated rehabilitation protocol may have constituted a novel situation of the sort that people high on openness to experience may find engaging. This possibility, of course, requires further investigation.

In contrast to previous research in which neuroticism and extraversion were associated with adherence to health behavior regimens $[6,8,11,12]$, neither neuroticism nor extraversion emerged as a predictor of adherence in the current study. The correlations between both personality dimensions and the two adherence measures were all in the predicted direction (i.e., negative for neuroticism and positive for extraversion), but were of insufficient magnitude to achieve statistical significance.

The current study had several key strengths. First, the sample was homogeneous with respect to injury and rehabilitation protocol, thereby ensuring the comparability across participants of the treatment for which adherence was assessed. Second, a prospective, longitudinal research design was used. By measuring the Big Five personality dimensions prior to surgery, it is possible to entertain an explanation of the findings that considers personality characteristics as potential contributors to the differences in adherence that were observed. Third, multiple measures were used to assess adherence to clinic-based rehabilitation activities. Because one of the measures was objective (attendance) and the other measure involved other-report (SIRAS), neither adherence index was hampered by the potential drawbacks of self-report (e.g., social desirability bias, forgetting).
Although the homogeneity of the sample is a strength of the study, it is also a limitation in that it is not possible to generalize the results to other sport injuries and even other protocols for the rehabilitation of the same injury. Further research with other injuries and rehabilitation protocols is needed. Another limitation of the study is that even though a prospective research design was used, the data are still correlational and no causal inferences can be drawn from the findings. Demonstration of causality may prove elusive, however, because it is not possible to manipulate personality experimentally. Also, even though neither of the two adherence measures involved self-report, the ceiling effect and restricted range of the attendance scores may have attenuated personality-adherence associations for that aspect of clinic-based rehabilitation, and the fact that the rehabilitation practitioners who completed the SIRAS for each participant were not masked with respect to the participants' attendance status may have biased the practitioners' adherence ratings. The difficulty with the attendance scores is not easily correctable, but the problem with the adherence ratings could be addressed in future research by having observers who are unaware of participants' attendance status complete the SIRAS for each participant.

In terms of implications for clinical practice, the findings suggest that knowledge of patients' personality may be useful in implementing accelerated rehabilitation protocols following ACL reconstruction. Knowing, for example, that a given patient is low in conscientiousness, agreeableness, or openness to experience may signal the need to provide added attention or motivation to increase the likelihood of that person adhering to the clinic-based aspects of rehabilitation program. Given that the Big Five personality dimensions accounted for 9 to 16 percent of the variance in attendance and rehabilitation professional adherence ratings, respectively, it is clear that a thorough understanding of adherence also requires consideration of a full array of situational, cognitive, and emotional factors [3]. Attending to and enhancing the adherence of people undergoing rehabilitation has the potential to improve treatment outcomes.

\section{CONFLICT OF INTEREST}

The authors confirm that this article content has no conflict of interest. 


\section{ACKNOWLEDGEMENTS}

Robert C. Hilliard is now at Ball State University. Allen E. Cornelius is now at the University of the Rockies. We gratefully acknowledge the cooperation of Joseph Sklar, M.D., Mark Pohlman, M.D., and John Corsetti, M.D. in conducting this research and thank Josh Avondoglio, Lisa Benjamin, Jeff Benoit, Kathy Bernardini, Jim Biron, Ruth Brennan-Morrey, John Brickner, Matt Buman, Judy Catalano, Tarra Cemborski, Kim Cochrane, Rosa Correa, Bob Crawford, Candi Daniele, Amie Dillman, Joann Golden, Reubin Gonzalez, Bryan Gross, Stephanie Habif, Whitney Hartmann, Jay Hatten, Carter Hunt, Jean Hutchinson, Angie Jensen, Aaron Kopish, Dawn Kresge, Ellie Laino, Amy Lowery, Kevin McAllister, Thomas Melvin, Alexa Mignano, Joe Monserrat, Liz Montemagni, Jonna Mullane, Rob Olenchak, Jens Omli, Scott Quarforth, Alice Robitaille, Raylene Ross, Carrie Scherzer, Josie Scibelli, Wendy Sewack, Ben Shachar, Sumiyo Shiina, and Marie Trombley for their assistance in data collection.

This research was supported in part by grant R29 AR44484 from the National Institute of Arthritis and Musculoskeletal and Skin Diseases. Its contents are solely the responsibility of the authors and do not represent the official views of the National Institute of Arthritis and Musculoskeletal and Skin Diseases.

\section{REFERENCES}

[1] Meichenbaum D, Turk DC. Facilitating treatment adherence: a practitioner's guidebook. New York, United States: Plenum 1987.

[2] Brewer BW. Adherence to sport injury rehabilitation regimens. In: Bull SJ, Ed. Adherence issues in sport and exercise. Chichester, England: Wiley 1999; pp. 145-68.

[3] Brewer BW. Psychological aspects of rehabilitation. In: Kolt GS, Andersen MB, Eds. Psychology in the physical and manual therapies. Edinburgh, Scotland: Churchill Livingstone 2004; pp. 39-53.

[4] Wiese-Bjornstal DM, Smith AM, Shaffer SM, Morrey MA. An integrated model of response to sport injury: psychological and sociological dynamics. J Appl Sport Psychol 1998; 10: 46-69.

[5] Costa PT, McCrae RR. The NEO Personality Inventory manual. Odessa, Florida, United States: Psychological Assessment Resources 1985.

[6] Booth-Kewley S, Vickers RR Jr. Associations between major domains of personality and health behavior. J Pers 1994; 62: 28198.

[7] Christensen AJ, Smith TW. Personality and patient adherence: correlates of the five-factor model in renal dialysis. J Behav Med 1995; 18: 305-13.
[8] Courneya KS, Hellsten LM. Personality correlates of exercise behaviour, motives, barriers, and preferences: an application of the five-factor model. Pers Individ Dif 1998; 24: 625-33.

[9] Saklofske DH, Austin EJ, Galloway J, Davidson K. Individual difference correlates of health related behaviours: preliminary evidence for links between emotional intelligence and coping. Pers Individ Dif 2007; 42: 491-502.

[10] Lin J, Chen L, Wang E, Cheng J. The relationship between extroversion and leisure motivation: evidence from fitness center participation. Soc Behav Pers 2007; 35: 1317-22.

[11] Saklofske DH, Austin EJ, Rohr BA, Andrews JJ. Personality, emotional intelligence, and exercise. J Health Psychol 2007; 12: 937-48.

[12] Wheeler K, Wagaman A, McCord D. Personality traits as predictors of adherence in adolescents with type I diabetes. J Child Adolesc Psychiatr Nurs 2012; 25: 66-74.

[13] Shelbourne KD, Nitz P. Accelerated rehabilitation after anterior cruciate ligament reconstruction. Am J Sports Med 1990; 18: 29299.

[14] Shelbourne KD, Klootwyk TE, DeCarlo MS. Update on accelerated rehabilitation after anterior cruciate ligament reconstruction. J Orthop Sports Phys Ther 1992; 15: 303-8.

[15] DeCarlo M, Klootwyk TE, Shelbourne KD. ACL surgery and accelerated rehabilitation: Revisited. J Sport Rehabil 1997; 6: 14456.

[16] Brewer BW, Van Raalte JL, Petitpas AJ, Sklar JH, Pohlman MH, Krushell RJ. Preliminary psychometric evaluation of a measure of adherence to clinic-based sport injury rehabilitation. Phys Ther Sport 2000; 1, 68-74.

[17] Brewer BW, Avondoglio JB, Cornelius AE, Van Raalte JL, Brickner JC, Petitpas AJ. Construct validity and inter-rater agreement of the Sport Injury Rehabilitation Adherence Scale. J Sport Rehabil 2002; 11: 170-8.

[18] Kolt GS, Brewer BW, Pizzari T, Schoo AMM, Garrett N. The Sport Injury Rehabilitation Scale: a reliable scale for use in clinical physiotherapy. Physiotherapy 2007; 93: 17-22.

[19] Costa PT, McCrae RR. Revised NEO Personality Inventory (NEO-PI) and NEO Five-Factor Inventory (NEO-FFI) professiona manual. Odessa, Florida, United States L: Psychological Assessment Resources 1992.

[20] Costa PT, McCrae RR, Dye DA. Facet scales for agreeableness and conscientiousness: a revision of the NEO Personality Inventory. Pers Individ Dif 1991; 12: 887-98.

[21] Costa PT, Somerfield MR, McCrae RR. Personality and coping: a reconceptualization. In: Zeidner M, Endler NS, Eds. Handbook of coping: theory, research, applications. New York, United States: Wiley 1996; pp. 44-61.

[22] Hogan J, Ones DS. Conscientiousness and integrity at work In: Hogan R, Johnson J, Briggs S, Eds. Handbook of personality psychology. San Diego, United States: Academic Press 1997; pp. 849-70.

[23] McCrae RR, John OP. An introduction to the five-factor model and its applications. J Pers 1992; 60: 175-215. 